\title{
Experimental Hemiplegia in the Monkey: Basal Ganglia Glucose Activity During Recovery
}

\author{
Sid Gilman, MD, George W. Dauth, PhD, Kirk A. Frey, MD, PhD, and John B. Penney, Jr, MD
}

\begin{abstract}
Unilateral ablation of cerebral cortical areas 4 and 6 of Brodmann in the macaque monkey results in a dense contralateral hemiplegia that recovers partially with time. During the phase of dense hemiplegia, the local cerebral metabolic rate for glucose (ICMRGIc) is decreased significantly in the caudate nucleus, putamen, globus pallidus, subthalamic nucleus, substantia nigra, and red nucleus of the hemisphere ipsilateral to the lesion. In the present study, ICMRGlc in the basal ganglia was studied during the phase of partial recovery of motor activity. ICMRGlc was partially restored, and the greatest degree of restoration occurred in structures with direct connections to the cerebral cortex (caudate nucleus, putamen, subthalamic nucleus, substantia nigra, and red nucleus). Restoration was least in structures that do not receive direct connections from the cerebral cortex (the internal and external segments of the globus pallidus). The findings support the hypothesis that corticofugal activity accounts for a substantial degree of functional recovery.
\end{abstract}

Gilman S, Dauth GW, Frey KA, Penney JB Jr: Experimental hemiplegia in the monkey: basal ganglia glucose activity during recovery. Ann Neurol 22:370-376, 1987

Ablation of areas 4 and 6 of Brodmann in the precentral cerebral cortex in the primate results immediately in a dense contralateral hypotonic hemiplegia $[6,8$, $12]$. With time, partial recovery of motor activity occurs in the affecred limbs $[8,12]$. The central nervous system structures and functions responsible for the recovery process have not been identified. We have undertaken studies of metabolic activity in the basal ganglia using 2-deoxyglucose to learn the metabolic response of these structures during the initial paralysis and subsequent recovery. One week after cerebral cortical ablation, the local cerebral metabolic rate for glucose (ICMRGlc) was significantly diminished in the ipsilateral caudate nucleus, putamen, globus pallidus, substantia nigra, and subthalamic nucleus [6]. We report the results of studies of ICMRGlc in the basal ganglia 8 weeks after ablation of cerebral cortical areas 4 and 6. At this time after ablation, motor function was partially restored in the affected limbs. The purpose of the present study was to determine the sites where changes in metabolic activity occur with time, as limb function becomes partially restored.

\section{Materials and Methods}

Six Macaca fascicularis monkeys weighing 2.5 to $3.5 \mathrm{~kg}$ were used for unilateral ablation of cerebral cortical areas 4 and 6 of Brodmann. The animals were examined neurologically to ensure that they did not have any motor impairment before the operation. The ablation was performed in the left cerebral hemisphere, as described previously [6, 12]. After recovery from the immediate effects of operation, the animals were examined neurologically every week [12]. The techniques used for deoxyglucose study were identical to those described previously [6]. Eight weeks after ablation, catheters were placed in the femoral vein and artery of the right leg with the animal under local anesthesia with lidocaine. The animals were restrained in a primate chair so that they could not move their limbs, and were kept in a quiet room for 1 hour. No attempt was made to interfere with vision or hearing. We used the technique for deoxyglucose autoradiography involving an intravenous bolus of $100 \mu \mathrm{Ci} / \mathrm{kg}$ of $\left[{ }^{14} \mathrm{C}\right] 2$-deoxyglucose in $1 \mathrm{ml}$ of saline, as described by Sokoloff et al $[19,20]$ and Dauth et al [6]. The brain was sectioned at $20 \mu \mathrm{m}$ on a cryostat. Three serial sections were taken every $400 \mu \mathrm{m}$ throughout the brain for autoradiographic analysis. The sections were placed on coverslips and dried rapidly on a hot plate. The sections and calibrated ${ }^{1 / 4} \mathrm{C}$ plastic standards were placed on Kodak SB5 $x$-ray film in a cassette and exposed for 6 days. Two $20-\mu \mathrm{m}$ serial sections adjacent to those used for autoradiography were processed for histological study [6]. One slide was stained with cresyl violet [18] and the other with $0.3 \%$ osmium tetroxide.

The autoradiograms were analyzed with a spot densitometer interfaced to a microcomputer that was programmed to convert film density values to glucose utilization rates using ${ }^{14} \mathrm{C}$ standards and plasma glucose and deoxyglucose curves $[5,6,20]$. The lumped and rate constants were those used by Kennedy and associates [15]. The glucose utilization rates
From the Department of Neurology, The Universiry of Michigan Medical School, Ann Arbor, MI 48109.

Received Oct 22, 1986, and in revised form Dec 29. Accepted for publication Feb 9, 1987.
Address correspondence to Dr Gilman, Department of Neurology, University of Michigan, 1914/0316 Taubman Health Center, 1500 E Medical Center Dr, Ann Arbor, MI 48109-0316. 
were determined for each structure every $400 \mu \mathrm{m}$ throughout the brain. At each level, the measurement consisted of the average of multiple readings from the three serial sections at that level. Each structure was measured separately, both ipsilateral and contralateral to the lesion. The final data values used in the analysis represented the average from all levels for a particular structure for each animal. The results of the statistical analyses were compared with data from a group of 6 unoperated control animals and a group of 6 animals with lesions of cerebral cortical areas 4 and 6 , examined 1 week after lesioning. The latter two groups were described previously [6].

Two statistical analysis procedures were used. First, a 2way repeated-measures analysis of variance (ANOVA) was applied to the data from each structure in the left and right hemispheres across the three groups. When indicated by significant $F$-ratios, an analysis of simple main effects was performed [21]. The second procedure was a 1-way ANOVA on the left-minus-right difference scores for each structure across the three groups. Significant $F$-ratios in the 1-way ANOVA led to Newman-Keuls tests for multiple pairwise comparisons [21].

The methods of statistical analysis used in these experiments require brief explanation. The 2-way repeated-measures ANOVA often requires the pooling of heterogeneous sources of variance in the error term for analysis of uncorrelated simple main effects which can bias the outcome of the test in an undetermined direction [21]. The analysis of correlated simple main effects for the left-versus-right hemisphere is not affected. Group differences could be inferred from the pattern of significant differences for the glucose utilization values of the left and right hemisphere for each group. However, we chose to use a 1-way ANOVA of difference scores and Newman-Keuls pairwise comparisons to provide a more direct analysis of between-group differences. This second analysis provides information on the betweengroup left-right differences, but not the absolute values for left and right hemispheres. These analytical methods are an attempt to detect substantial group effects on global metabolic rates, while minimizing the number of animals needed to demonstrate significant alterations in lCMRGlc.

\section{Results}

\section{Clinical Observations}

Details of the clinical effects of unilateral ablation of areas 4 and 6 of Brodmann in the macaque monkey are described elsewhere $[8,12]$. The present set of animals all showed a dense right hemiplegia upon recovery from anesthesia. During the third week after operation, the right limbs developed increased resistance to passive manipulation, consisting of a mild plastic response to flexion or extension at the right shoulder, elbow, wrist, and knee. The deep tendon reflexes of the right limbs increased, so that they were larger in amplitude than those of the left limbs. In the fourth week, the right arm developed flexion movements at the shoulder and elbow in association with reaching movements of the left limbs. The right limbs developed greater resistance to passive manipulation, and the deep tendon reflexes in these limbs became even more enhanced. During the sixth week, the right arm extended and the right hand made crude contact with a reflex hammer, coincident with the left hand, when the animal's chest was struck lightly with the hammer. In the eighth week, with the animal held in the vertical position from the chest, the right arm flexed 90 degrees and the right leg extended. Ambulation was performed with the left arm and leg and the right leg, with the right arm only occasionally participating. The animal usually reached for objects with the left limbs alone, although the right arm occasionally participated with incomplete extension movements. The increased resistance to passive manipulation and enhanced deep tendon reflexes persisted in the right limbs. Orienting responses to light cutaneous contact did not recover in the right limbs. The degree of clinical recovery was essentially the same in the entire set of animals.

\section{Histological Results}

The histopathological changes were equivalent in all the animals. The cortical areas involved by the lesions were similar to those in the animals that had been studied histologically 1 week after ablation [6]. The lesions involved nearly all of the agranular isocortex, sparing the extreme medial portion of the prefrontal gyrus and the depths of the superior precentral sulcus. There was demyelination in the white matter underlying the lesion and in the ipsilateral internal capsule, crus cerebri, and medullary pyramid. The areas of demyelination under the lesion did not directly involve the caudate nucleus or putamen (Fig 1). No edema was present. Ipsilateral to the lesion were infiltrates of $\mathrm{mi}$ croglial cells and astrocytes in the putamen, caudate nucleus, subthalamic nucleus, and rostral red nucleus, and in the ventral anterior, ventral lateral, and centromedian nuclei of the thalamus. There was slight shrinkage of the putamen, and a slight increase in the packing density of the neurons. No infiltrate could be identified in the pons. The large neurons of the ventral anterior and lateral nuclei of the thalamus were absent. There were no histological changes in the globus pallidus or substantia nigra.

\section{Local Cerebral Metabolic Rates for Glucose}

The results of the 2-way repeated-measures ANOVA for left-right comparisons in the 8-week group are presented in Table 1. ICMRGlc was significantly less in the left hemisphere compared to the right for the caudate nucleus, putamen, globus pallidus, subthalamic nucleus, and red nucleus (Fig 2D-F). The differences between groups are illustrated in Figure 3, which shows the pattern of changes in ICMRGlc relative to control at 1 and 8 weeks after ablation. The predominant effects are in the left (ablated) hemisphere, with 


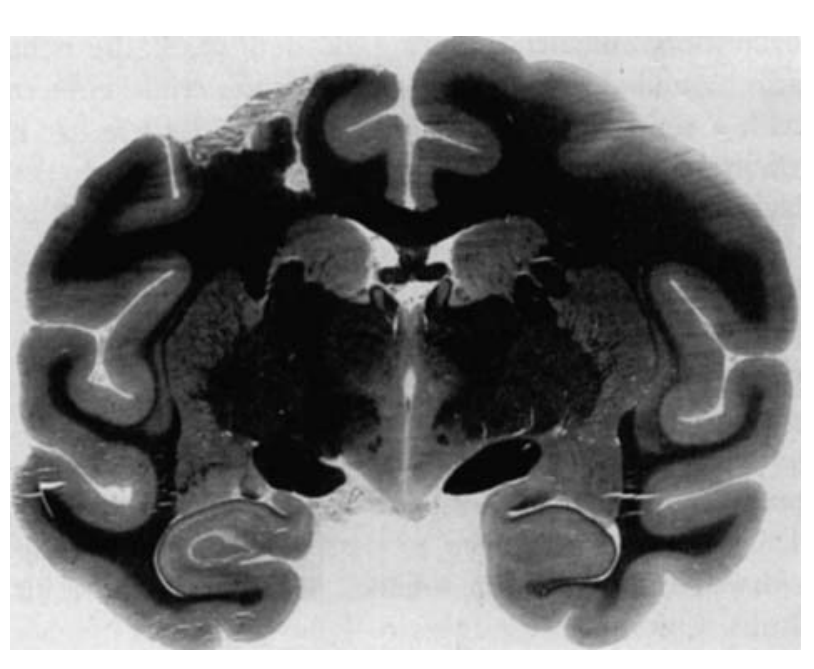

Fig 1. Osmium-stained section from a monkey 8 weeks after unilateral ablation of cerebral cortical areas 4 and 6 of Brodmann. There is no pallor in the white matter surrounding the ablation, indicating that edema bas resolved. There is no evidence of edema in the subcortical gray matter.

moderate to substantial reductions in the basal ganglia at 1 week (Fig 2A-C) and variable recovery at 8 weeks (Fig 2D-F). In the left hemisphere, at 1 week after the ablation the caudal portions of the caudate nucleus and putamen (caudal to the anterior commissure) have lower metabolic rates than the rostral portions (rostral to the anterior commissure). The same relationship is maintained at 8 weeks, even though there is substantial recovery of ICMRGlc at this time (Fig 3). In the globus pallidus, ICMRGIc is reduced substantially at 1 week and only marginal recovery occurs at 8 weeks. In the red nucleus, metabolic activity is decreased markedly at 1 week and moderate recovery takes place by 8 weeks. In the substantia nigra, there is a moderate reduction at 1 week and almost complete recovery by 8 weeks.

In the right hemisphere, a small to moderate reduction in ICMRGic occurs at 1 week in the caudate nucleus, putamen, and globus pallidus. At 8 weeks, there is almost complete recovery in the caudate nucleus and putamen and only marginal recovery in the globus pallidus (Fig 3). In the subthalamic nucleus, there is a modest reduction in ICMRGic on the left side at 1 week, but a bilateral increase above control levels at 8 weeks.

The left-right difference scores for control animals and animals 1 week and 8 weeks after ablation are presented in Table 2, along with the results of the 1-way ANOVA on the three groups. There are significant group differences in the caudate nucleus, putamen, globus pallidus, subthalamic nucleus, red nucleus, and substantia nigra pars reticulata, but not in the substantia nigra pars compacta. Pairwise com-
Table 1. ICMRGlc 8 Weekr after Ablation of Cerebral Cortical Areas 4 and 6 of Brodmann ${ }^{2}$

\begin{tabular}{llll}
\hline Structure & Left & Right & L/R \\
\hline CD-r & $30.62(2.41)$ & $31.53(2.30)$ & $0.97^{\mathrm{b}}$ \\
CD-c & $32.99(2.69)$ & $35.12(2.40)$ & $0.94^{\mathrm{b}}$ \\
PUT-r & $28.27(2.82)$ & $32.17(2.45)$ & $0.89^{\mathrm{c}}$ \\
PUT-c & $28.59(3.53)$ & $35.37(3.02)$ & $0.81^{\mathrm{c}}$ \\
GPE & $13.63(1.66)$ & $15.45(1.60)$ & $0.88^{\mathrm{c}}$ \\
GPI & $13.03(1.39)$ & $16.57(1.69)$ & $0.79^{\mathrm{c}}$ \\
NSTH & $34.41(3.45)$ & $39.66(4.38)$ & $0.87^{\mathrm{c}}$ \\
SNR & $19.56(3.06)$ & $21.00(3.01)$ & 0.93 \\
SNC & $27.61(2.99)$ & $29.13(3.02)$ & 0.95 \\
RN & $24.25(3.52)$ & $29.39(3.04)$ & $0.83^{\mathrm{b}}$ \\
SEPT & $17.14(1.92)$ & $17.19(1.94)$ & 1.00 \\
LGN & $26.71(3.21)$ & $27.20(2.86)$ & 0.98 \\
MGN & $45.44(3.93)$ & $45.90(3.52)$ & 0.99 \\
\hline
\end{tabular}

avalues are mean (SEM) $\mu \mathrm{mol} / 100 \mathrm{gm} / \mathrm{minute}$.

${ }^{\text {b }}$ Significant left versus right comparison; $p<0.05$.

'Significant left versus right comparison; $p<0.01$.

ICMRGIc = local cerebral metabolic rate for glucose; $\mathrm{CD}=$ caudate nucleus; $\mathrm{r}=$ measured rostral to the anterior commissure; $\mathrm{c}=$ measured between the anterior commissure and the red nucleus; PUT $=$ putamen; GPE = globus pallidus external segment; GPI = globus pallidus internal segment; NSTH $=$ subthalamic nucleus SNR = substantia nigra pars reticulata; $S N C=$ substantia nigra pars compacta; $R N=$ red nucleus; $S E P T=$ septal nuclei; $L G N=$ lateral geniculate nucleus; $\mathrm{MGN}=$ medial geniculate nucleus.

parisons of difference scores reveal that by 8 weeks there was complete correction of the asymmetry in ICMRGIc in the rostral caudate nucleus, red nucleus, and substantia nigra pars reticulata (Table 3). This is indicated by the pattern of significant and nonsignificant pairwise comparisons for these structures. For example, in the case of the rostral caudate nucleus, the comparisons of control versus 1-week groups and 1-week versus 8-week groups are both significant, but there is no significant difference between the control and 8-week groups (Table 3). Conversely, all three comparisons are significant for the caudal caudate nucleus and both parts of the putamen, indicating that there is only partial correction of the asymmetry in ICMRGlc in these structures. The pattern of significant pairwise comparisons of both portions of the globus pallidus indicates that there is little if any correction of the asymmetry at 1 week.

The subthalamic nucleus is an interesting and special case. At 1 week there is an asymmetry in this nucleus, with reduced values on the left compared to the right. By 8 weeks, both sides show an elevated ICMRGlc compared to control values, though the asymmetry is the same but more pronounced than at 1 week (Fig 3). The pattern of significant pairwise comparisons for the subthalamic nucleus supports this observation (Table 3). Finally, although there are no significant left-versusright or between-group differences for the septal nuclei, there appears to be a bilateral reduction in 

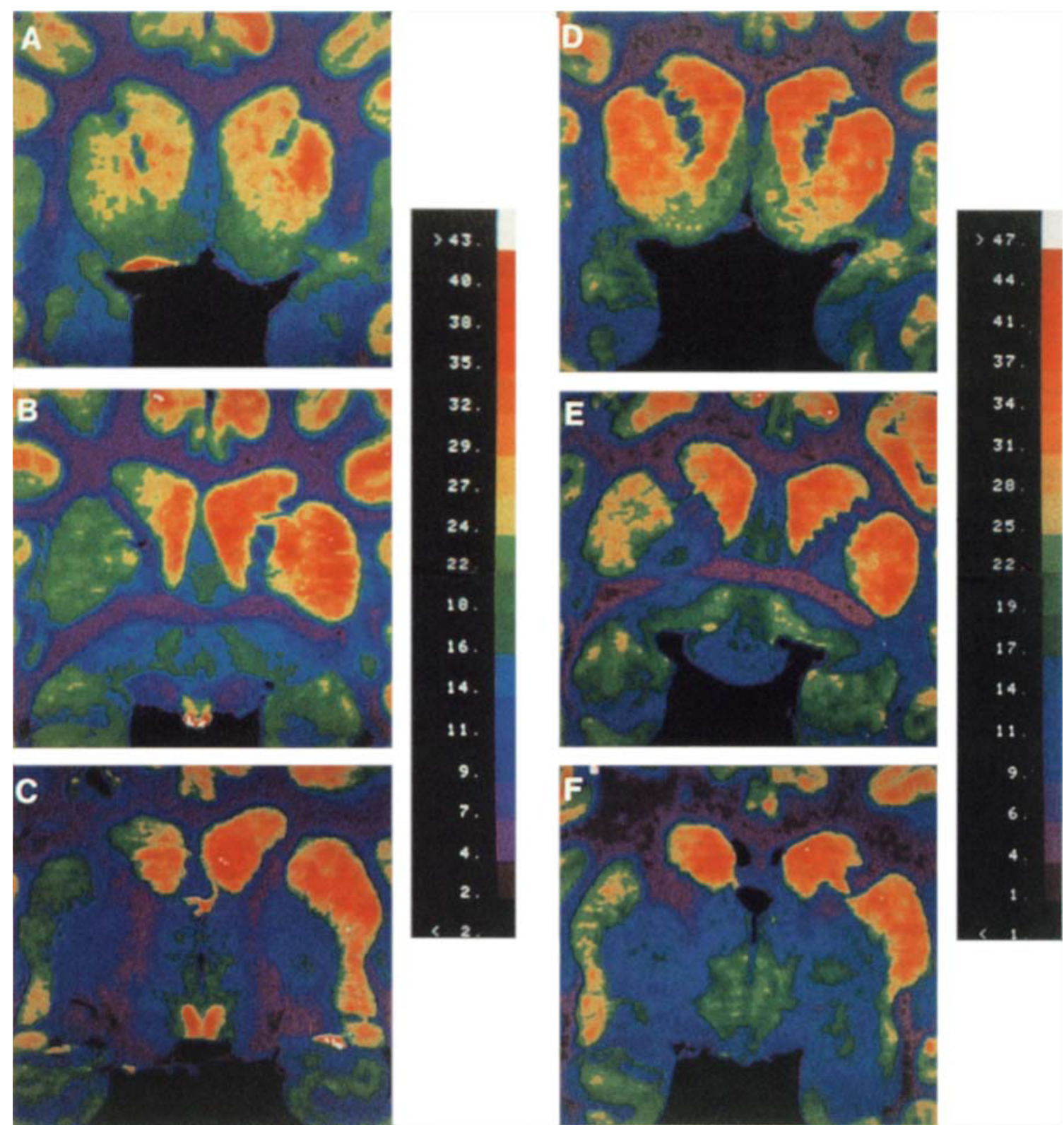

Fig 2. Computer-enhanced deoxyglucose autoradiograms from 2 monkeys with ablations of cerebral cortical areas 4 and 6 of Brodmann. (A-C) From an animal 1 week after the ablation. (D-F) From an animal 8 weeks after ablation. The color bars for each animal are calibrated for the local cerebral metabolic rate for glucose at $\mu \mathrm{mol} / 100 \mathrm{gm} / \mathrm{min}$.

ICMRGlc at 1 week and bilateral partial recovery at 8 weeks (Fig 3). The data for the septal nuclei include both the medial and lateral nuclei.

\section{Discussion}

The present study supplements our previous observations on animals 1 week after ablation of areas 4 and 6 of Brodmann [6] by demonstrating at 8 weeks persistent asymmetries in ICMRGlc in structures receiving

direct connections from the cerebral cortex. At 1 week, ICMRGlc was significantly less on the left compared to the right in the caudate nucleus, putamen, globus pallidus, subthalamic nucleus, substantia nigra, and red nucleus [6]. In the present study there is evidence of recovery, but the ICMRGlc remains depressed in the left caudate nucleus, putamen, globus pallidus, and red nucleus. Metabolic activity in the subthalamic nucleus is less on the left than on the right, but both sides show values that are elevated above control levels. There appears to be complete recovery in the substantia nigra at 8 weeks (Table 1, Fig 3).

The degree of recovery in ICMRGic at 8 weeks is variable, but it is greatest in the structures that receive 


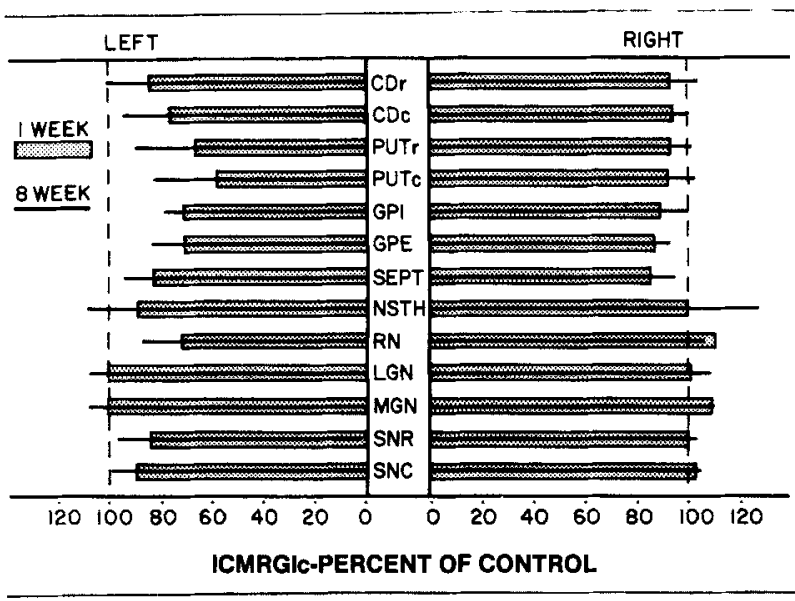

Fig 3. Local cerebral metabolic rate for glucose (lCMRGlc) for animals with unilateral ablations of cerebral cortical areas 4 and 6 of Brodmann at 1 and 8 weeks after ablation, expressed as a percentage of the corresponding structures in unoperated control animals. The percentages were calculated based on the mean ICMRGlc for each of the three groups. (See Table 1 for abbreviations.)

Table 2. ICMRGlc Left-Right Difference Scores ${ }^{\mathrm{a}}$

\begin{tabular}{lrrl}
\hline Structure & \multicolumn{1}{l}{ Control } & \multicolumn{1}{l}{ 1-Week } & 8-Week \\
\hline CD-r & $-0.18(0.11)$ & $-2.35(0.54)$ & $-0.91(0.42)^{\mathrm{c}}$ \\
CD-c & $0.53(0.48)$ & $-6.22(1.22)$ & $-2.13(0.66)^{\mathrm{c}}$ \\
PUT-r & $-0.08(0.38)$ & $-8.20(1.01)$ & $-3.46(1.01)^{\mathrm{c}}$ \\
PUT-c & $0.35(0.51)$ & $-11.70(1.61)$ & $-6.78(0.84)^{\mathrm{c}}$ \\
GPE & $-0.08(0.33)$ & $-2.48(0.50)$ & $-1.82(0.27)^{\mathrm{c}}$ \\
GPI & $-0.45(0.54)$ & $-2.82(0.37)$ & $-3.54(0.64)^{\mathrm{c}}$ \\
NSTH & $-0.72(0.65)$ & $-3.63(1.07)$ & $-5.25(1.56)^{\mathrm{b}}$ \\
SNR & $0.46(0.50)$ & $-3.10(1.19)$ & $-1.44(0.44)^{\mathrm{b}}$ \\
SNC & $-0.64(0.55)$ & $-3.83(1.49)$ & $-1.51(0.43)$ \\
RN & $-0.20(0.31)$ & $-10.70(2.81)$ & $-5.14(0.60)^{\mathrm{c}}$ \\
SEPT & $-0.08(0.36)$ & $-0.32(0.12)$ & $-0.05(0.10)$ \\
LGN & $-0.45(0.40)$ & $-0.12(0.65)$ & $-0.49(0.64)$ \\
MGN & $1.77(1.03)$ & $-3.38(1.89)$ & $-0.46(1.31)$ \\
\hline
\end{tabular}

${ }^{a}$ Values are mean $(\mathrm{SEM}) \mu \mathrm{mol} / 100 \mathrm{gm} /$ minute.

bSignificant 1-way ANOVA; $p<0.05$.

'Significant 1-way ANOVA; $p<0.01$

See Table 1 for abbreviations.

direct connections from the cortex. Thus, the degree of recovery in the left hemisphere is greatest in the caudate nucleus and putamen, and least in the globus pallidus. Permanent loss of large numbers of corticofugai fibers projecting directly into these sites must account for the sustained decrease in metabolic activity in the caudate nucleus and putamen. Glucose utilization rates at 8 weeks also remain substantially depressed in the globus pallidus, which does not receive direct connections from the cerebral cortex. The effect upon the globus pallidus is indirect, evidently resulting from a change in synaptic activity mediated via the caudate nucleus, putamen, and possibly other nuclei,
Table 3. Pairwise Comparisons of lCMRGlc

Left-Right Difference Scores

\begin{tabular}{llll}
\hline & $\begin{array}{l}\text { Control } \\
\text { vs. }\end{array}$ & $\begin{array}{l}\text { Control } \\
\text { vs. }\end{array}$ & $\begin{array}{l}1 \text {-Week } \\
\text { vs. }\end{array}$ \\
Structure & 1 -Week & 8 -Week & 8 -Week \\
\hline CD-r & $*$ & NS & $*$ \\
CD-c & $*$ & $*$ & $*$ \\
PUT-r & $*$ & $*$ & $*$ \\
PUT-c & $*$ & $*$ & $*$ \\
GPE & $*$ & $*$ & NS \\
GPI & $*$ & $*$ & NS \\
NSTH & NS & $*$ & NS \\
SNR & $*$ & NS & $*$ \\
SNC & $\ldots$ & $\ldots$ & $\ldots$ \\
RN & $*$ & NS & $*$ \\
SEPT & $\ldots$ & $\ldots$ & $\ldots$ \\
LGN & $\ldots$ & $\ldots$ & $\ldots$ \\
MGN & $\ldots$ & $\ldots$ & $\ldots$ \\
\hline
\end{tabular}

* = significant Newman-Keuls pairwise comparison; NS = nonsignificant pairwise comparison; ... = not tested, 1-way ANOVA not significant.

See Table 1 for abbreviations.

such as the subthalamic nucleus, which receive input from ablated regions of cerebral cortex and project into the globus pallidus.

The restoration of ICMRGlc in the caudate nucleus and putamen could result from a variety of mechanisms, including: increased activity of undamaged corticofugal connections; restored activity in corticofugal projections that are injured, but not destroyed, in the initial ablation; enhanced activity of local interneuronal networks within the nuclei; heightened activity of projections from subcortical sites to these nuclei; sprouting of fibers from undamaged axons within the nucle or projecting to the nuclei from other sites [10]; and activity of infiltrative glial cells $[1,4,11]$. While we cannot currently differentiate among these possibilities, our findings lend support to the hypothesis that enhanced corticofugal activity from preserved cortical areas may account for a substantial amount of the recovery of lCMRGlc seen at 8 weeks.

Projections from cerebral cortical area 9 to the basal ganglia overlap with those from area 6 , and projections from areas 3,1, and 2 overlap with those from area 4 $[3,7,13,14,16,17]$. Area 5 also sends projections into the basal ganglia that overlap with those from area 4 [7]. Eight weeks after ablation, both the rostral and caudal portions of the putamen recover to about the same degree, whereas the rostral portion of the caudate nucleus recovers to a greater degree than the caudal portion. The reason for this may be related to the density of corticofugal projections to the caudate nucleus compared to the putamen and other structures. Much of the input to the caudate nucleus arises 
in the prefrontal cortex, particularly area 9, and to some extent areas 6 and 4 . The latter two areas project more densely to the putamen than to the caudate nucleus. Thus, the initial decrease of metabolic activity in the caudate nucleus at 1 week after ablation was relatively less than that in the putamen, corresponding to the greater density of projections from areas 4 and 6 of cerebral cortex to the putamen than to the caudate nucleus. During the recovery process, the unablated neurons in area 9 could account for a greater enhancement of metabolic activity in the rostral portion of the caudate nucleus than in the caudal portion or in either portion of the putamen.

Without further study we cannot determine the extent to which gliosis contributes to the "recovery" of ICMRGlc in the caudate nucleus, putamen, and other structures that receive projections directly from the ablated cortex. The macrophage and microglial reaction in gray matter areas that receive direct cortical input was less prominent in the 8-week animals than it was in the animals killed 1 week after ablation [6]. There was a more prominent astrocytic reaction at 8 weeks, and shrinkage became apparent only at 8 weeks. The histological changes at 1 and 8 weeks were more apparent in the putamen than in the caudate nucleus. The globus pallidus, which does not receive projections from areas 4 and 6 , had no histological changes at either time point. Likewise, the substantia nigra showed no histological changes at either time point, although it has been reported that area 6 projects to the substantia nigra [16]. Thus, we can rule out gliosis as a contributory factor in the alteration of ICMRGIc in structures that do not receive projections from the ablated cortex, such as the globus pallidus.

In both a previous study [6] and the present one, we found no statistically significant effect of the precentral ablation on ICMRGlc in the septal region, but the findings suggest that 1CMRGlc is suppressed in the septal region bilaterally at 1 week after ablation and is partially recovered at 8 weeks (Fig 3). Since there are no direct projections from areas 4 and 6 to the septal region, effects from the ablation are probably mediated via prefrontal or cingulate cortex, which have reciprocal projections to the septal region [2]. Another possible explanation is that our lesioning of areas 4 and 6 included the cerebral cortex along the mesial surface of the hemisphere and may have damaged some efferents from the cingulate gyrus (Fig 1).

Findings compatible with ours have been reported by Deuel and Collins $[9,10]$, who studied the effects of lesions of the frontal association cortex on sensory neglect and ICMRGlc in monkeys. While the animals exhibited neglect, ICMRdic was decreased ipsilateral to the cortical lesion in the caudate nucleus, putamen, and globus pallidus, and in some other cortical and thalamic structures. After animals recovered from ne- glect, there was almost complete recovery of 1CMRGlc in the caudate nucleus, putamen, and globus pallidus. The findings from the studies of Deuel and Collins $[9$, 10] differ from the present findings with respect to the area of cerebral cortex ablated and the fact that the survival time of the recovered animals was variable ( 4 to 10 weeks). In Deuel and Collins's study, the animals in the recovery group were not studied with deoxyghucose until behavioral recovery was complete. In our studies, the deoxyglucose experiments were performed at fixed time points ( 1 or 8 weeks after the ablation). The animals at 8 weeks showed incomplete recovery from hemiplegia as defined by clinical examination. The size and location of the cortical lesion and the lack of full clinical recovery may account for the incomplete recovery of ICMRGlc in our studies. Preliminary unpublished data from our laboratory indicate that substantial behavioral recovery can occur after ablations of areas 4 and 6 , but the recovery may take as long as 6 months. We expect to see more complete recovery of ICMRGlc in animals with motor cortex ablations at the longer survival times, when the animals show better behavioral recovery.

Supported in part by Grant NS 19613 from the National Institute of Neurological and Communicative Disorders and Stroke and Grant R-231-84 from the United Cerebral Palsy Educational and Research Foundation, Inc. Dr Frey was a trainee on Grant GM 07863 from the National Institutes of Health.

\section{References}

1. Agranoff BW, Frey KA: A regional metabolic contrast method for the study of brain pathology. Ann Neurol 15(suppl):S93S97, 1984

2. Brodal A: Neurological Anatomy, ed 3. New York, Oxford Universiry Press, 1981

3. Carpenter MB: Anatomy of the corpus striatum and brain stem integrating systems. In Brooks VB (ed): Handbook of Physiology, The Nervous System, Vol II, Part 2. Bethesda, American Physiological Society, 1981, pp 947-995

4. Cooper RM, Thurlow GA, Rooney BJ: 2-Deoxyglucose uptake and histologic changes in rat thalamus after neocortical ablations. Exp Neurol 83:134-143, 1984

5. Dauth GW, Frey KA, Gilman S: A densitometer for quantitative autoradiography. J Neurosci Methods 9:243-251, 1983

6. Dauth GW, Gilman S, Frey KA, Penney JB Jr: Basal ganglia glucose utilization after recent precentral ablation in the monkey. Ann Neurol 17:431-438, 1985

7. DeLong MR, Georgopoulos AP: Motor functions of the basal ganglia. In Brooks VB (ed): Handbook of Physiology, The Nervous System, Vol II, Part 2. Bethesda, American Physiological Society, 1981, pp 1017-1061

8. Denny-Brown D: The Cerebral Control of Movement. Liverpool, Liverpool University Press, 1966

9. Deuel RK, Collins RC: Recovery from unilateral neglect. Exp Neurol 81:733-748, 1983

10. Deuel RK, Collins RC: The functional anatomy of frontal lobe 
neglect in the monkey: behavioral and quantitative 2-deoxyglucose studies. Ann Neurol 15:521-529, 1984

11. Frey KA, Agranoff BW: Barbiturate-enhanced detection of brain lesions by carbon-14-labeled 2-deoxyglucose autoradiography. Science 219:879-881, 1983

12. Gilman S, Lieberman JS, Marco LA: Spinal mechanisms underlying the effects of unilateral abiation of areas 4 and 6 in monkeys. Brain 97:49-64, 1974

13. Goldman PS, Nauta WJH: An intricately patterned prefrontocaudate projection in the rhesus monkey. J Comp Neurol 171:369-385, 1977

14. Goldman-Rakic PS: Cytoarchitectonic heterogeneity of the primate neostriatum: subdivision into island and matrix cellular compartments. J Comp Neurol 205:398-413, 1982

15. Kennedy C, Sakurada O, Shinohara M, et al: Local cerebral glucose utilization in the normal conscious macaque monkey. Ann Neurol 4:293-301, 1978

16. Künzle H: An autoradiographic analysis of the efferent connections from premotor and adjacent prefrontal regions (areas 6 and 9) in Macaca fascicularis. Brain Behav Evol 15:185-234, 1978

17. Künzle H: Bilateral projections from precentral motor cortex to the putamen and other parts of the basal ganglia. An autoradiographic study in Macaca fascicularis. Brain Res 88:195-209, 1975

18. Luna LG: Manual of Histologic Staining Methods of the Armed Forces Institute of Pathology, ed 3. New York, McGraw-Hill, 1968

19. Sokoloff $L$ : Localization of functional activity in the central nervous system by measurement of glucose utilization with radioactive deoxyglucose. J Cereb Blood Flow Metab 1:7-36, 1981

20. Sokoloff L, Reivich M, Kennedy $C$, et al: The $\left[{ }^{14} \mathrm{C}\right] \mathrm{de}-$ oxyglucose method for the measurement of local cerebral glucose utilization: theory, procedure and normal values in the conscious and anesthetized albino rat. J Neurochem 28:897916, 1977

21. Winer BJ: Statistical Principles in Experimental Design. New York, McGraw-Hill, 1967 